
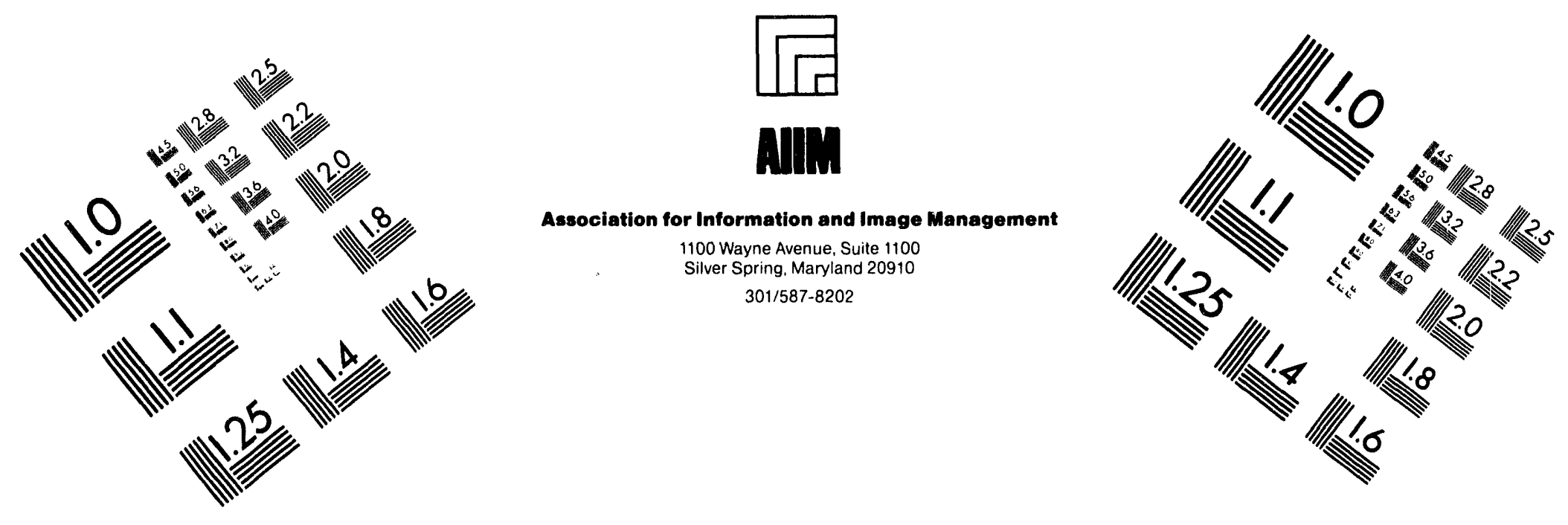

Centimeter

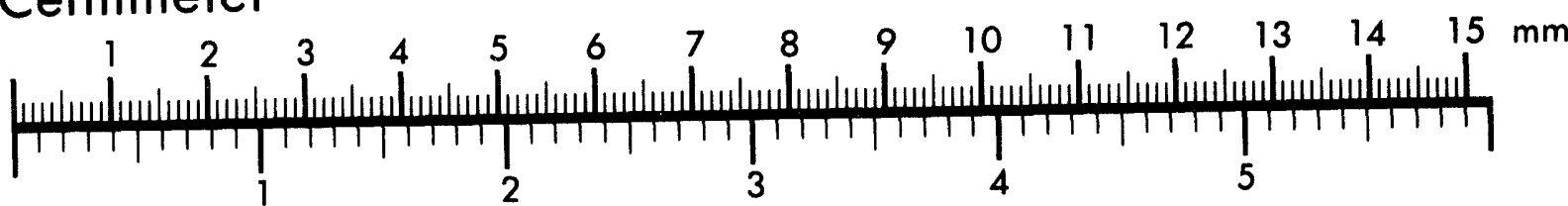
Inches
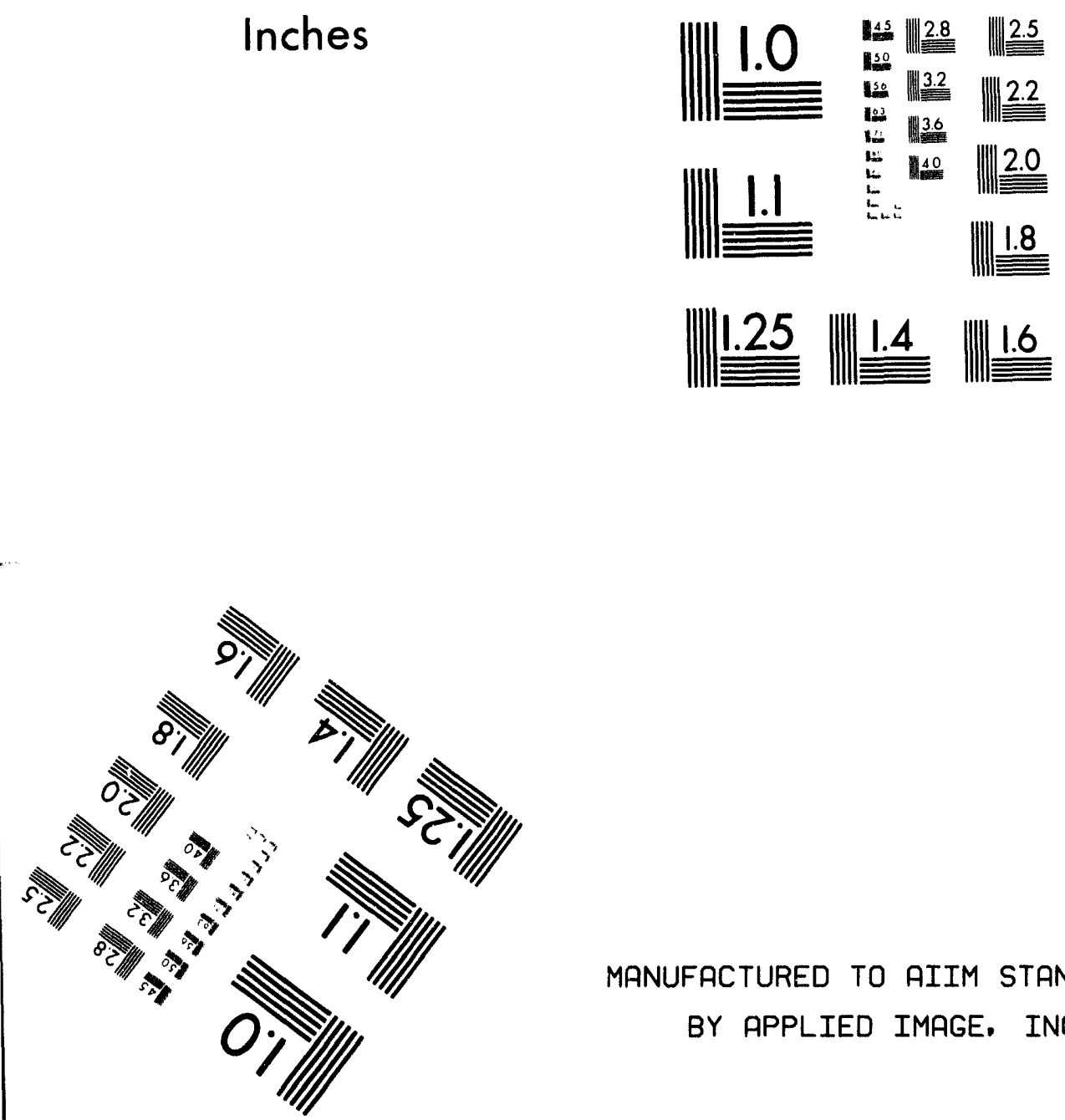

MANUFACTURED TO AIIM STANDARDS

BY APPLIED IMAGE, INC.

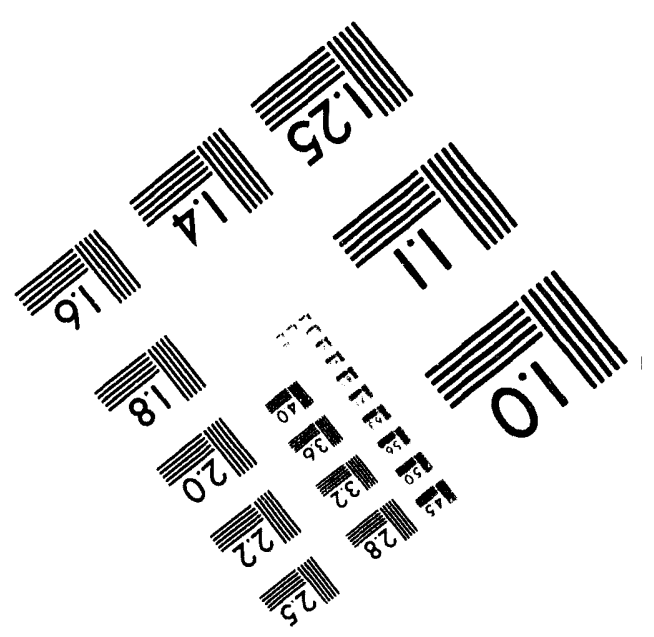



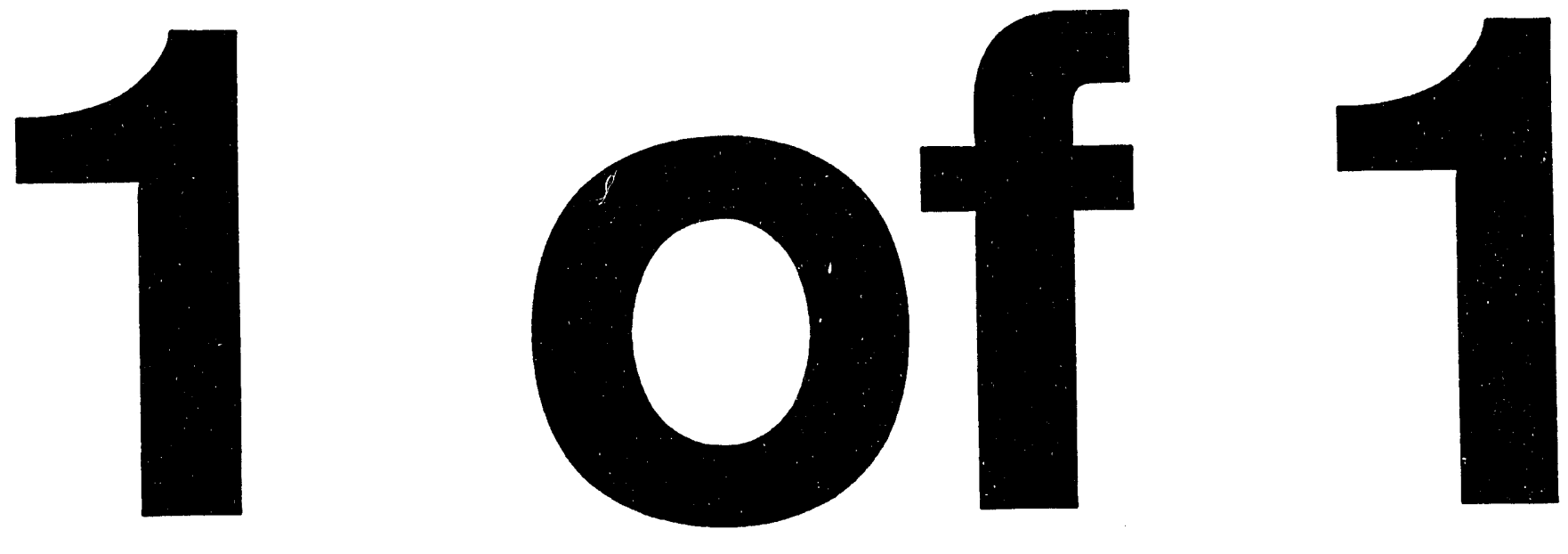


\title{
UV Laser-Surface Interactions Relevant to Analytic Spectroscopy of Wide Band Gap Materials
}

\author{
DOE Contract DE-FG06-92ER14252
}

\author{
P.I.: J. Thomas Dickinson \\ Department of Physics \\ Washington State University \\ Pullman, WA 99164-2814
}

\author{
Contract Monitor: Dr. Clem Yonker \\ BES-Chemical Sciences Division
}

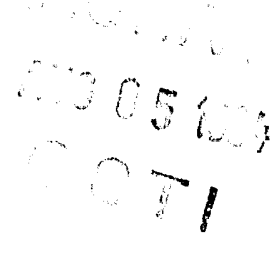

Laser ablation has important applications in materials analysis, particularly for refractory materials and for fast screening environmental analysis. We have been examining the details of processes that lead to the emission and formation of particles (atomic/molecular ground state neutrals, excited neutrals, tions, electrons) when materials are irradiated with pulsed UV laser light. Our studies bear directly on achieving understanding and control of emission intensities and particle characteristics for use in materials analysis, particularly of wide bandgap materials. These materials are often difficult to transport into the gas phase for analysis and in a number of high technology applications, such as laser

Our work has concentrated on the study of the desorption and ablation of material from nominally transparent, wide bandgap materials by UV excimer radiation at modest fluences which depends upon the presence of strongly absorbing lattice defects. In MgO, photo-electronic processes are not capable of generating such defects, so that $\mathrm{MgO}\left(\mathrm{E}_{\mathrm{g}}=\right.$ $7.9 \mathrm{eV})$ is highly resistant to radiolysis by UV photon and ( $<300 \mathrm{keV}$ ) electron bombardment. However, strongly absorbing defects can be produced mechanically by dislocation motion during deformation. These defects may be produced prior to laser exposure during sample preparation (e.g., cleaving or polishing $1,2,3$ ). We have shown that sustained ablation can be achieved at fluences where the thermal stresses accompanying pulsed laser irradiation deform the material and produce sufficient numbers of defects to replace those destroyed by emission processes and thermal annealing. At lower fluences, pre-existing impurities and defects control absorption and thus the response to laser irradiation. These interactions are the key to the understanding of incubation effects, where high-fluence laser pulses interact with pre-existing defects to gradually increase defect densities, ultimately leading to damage and sustained ablation.

Because of the strong UV absorption provided by mechanically produced defects in $\mathrm{MgO}$, cleaved surfaces are often much more resistant to UV interactions than polished surfaces. We have been able to show that the point-to-point variation in the response of cleaved surfaces can be quite significant due to localized deformation during cleavage. ${ }^{4}$ This leads, for example, the production of zones of a few microns exhibiting localized melting and hole formation. We have recently demonstrated a luminescence technique for imaging variations in defect densities at fluences well below the threshold for sustained ablation. We observe, for example, high concentrations of luminescent defects along cleavage steps, consistent with previous observations of strong laser interactions along cleavage steps under high fluence irradiation. In addition, materials that have been polished, abraded, indented, or repeatedly irradiated with pulses of excimer laser light, exhibit this highly localized photoluminescence. Enhanced defect densities along cleavage steps were directly verified by imaging the laser-induced photoluminescence due to these defects. ${ }^{5}$ Cleaved samples were irradiated in air with low fluence (unfocused) excimer laser radiation at $248 \mathrm{~nm}$. Illumination of deformed $\mathrm{MgO}$ produces a violet-blue luminescence (peak $\sim 410 \mathrm{~nm}$ ), due to the excitation of high order vacancy complexes (probably dominated by oxygen vacancies) produced by deformation. 6 The sample was positioned in the focal plane of a Zeiss stereomicroscope equipped with a $35-\mathrm{mm}$ camera.

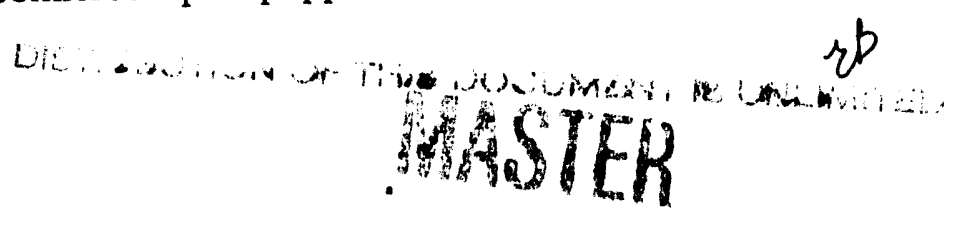


The photoluminescence is photographed with high speed black-and-white and color film (ASA 3200), the latter yielding blue images. Photographs taken under normal, white-light illumination and SEM micrographs can be compared with time exposures of the photoluminescence to identify the surface features responsible for the observed luminescence.

At fluences too low to maintain a sustained ablative response, defect-mediated neutral emissions often accompany the first few laser pulses on previously unexposed surfaces. However, with exposure to repeated pulses the relevant defects are depleted and emission gradually ceases. In this range of fluences, surface segregation of impurities can have dramatic effects on the composition of emitted neutral atoms and molecules. For instance, neutral emissions from (carbon) arc-fused $\mathrm{MgO}$ during the first few pulses of laser radiation are typically deficient in atomic and molecular oxygen. We have made recent measurements showing that virtually all oxygen emission under these conditions occurs in the form of carbon oxides.

In the case of ionic materials like $\mathrm{MgO}$, we show that defect-mediated ion emission occurs at low fluences by Coulomb repulsion. As in the case of defect-mediated neutral emissions, ion emission depletes the supply of relevant defects (at low fluences), so that the emission accompanying successive pulses gradually decreases and eventually ceases. We can show that the kinetic energies of $\mathrm{Mg}^{+}$and $\mathrm{Mg}^{2+}$ ions from $\mathrm{MgO}$ at fluences of about $1 \mathrm{~J} / \mathrm{cm}^{2}$ are consistent with Coulomb repulsion due to the ionization of nearby lattice defects (probably surface F-centers). We are currently taking angular distribution measurements of the positive ion emission trajectories that indicate this ion emission is strongly directed along the surface normal (observed full width of $5^{\circ}= \pm 2.5^{\circ}$ ); this requires that these ions are ejected from surface sites directly atop ionizable defects.

We are also acquiring ion angular distributions on polycrystalline $\mathrm{MgO}$ thin films deposited on a $\mathrm{Si}(111)$ wafer by laser ablation deposition in vacuum. Ion microprobe analysis of these films indicate that they are significantly deficient in oxygen (composition $\mathrm{MgO} \sim 0.8$ ); therefore these films necessarily have very high densities of F-centers (oxygen vacancies). The angular distributions from the films are similar to those from the polished samples, with the exception that the "core" region of the distribution shows structure which we attribute to emission from crystalline grains with slightly different orientations. However, ion emissions from the films are much more intense than the emissions from the polished $\mathrm{MgO}$ single crystals, consistent with an emission mechanism involving high densities of surface F-centers. These observations suggest that ion intensity measurements at low fluences serves as a quantitative measure of defect densities on such surfaces.

Work has recently been initiated on the desorption and ablation of other materials which are directly related to environmental problems at $\lambda=248 \mathrm{~nm}$ incident light. We are examining the resulting plume fluorescence for single crystal $\mathrm{NaNO}_{3}$, (a principle constituent of Hanford Tank materials) calcite (an important soil component), pulverized pure quartz (sand and soil component), pulverized pure quartz mixed with $\mathrm{Cr}, \mathrm{Eu}$, and $\mathrm{U}$ containing compounds, and a Hanford Soil with and without heavy metals added. We are determining the conditions conducive for obtaining atomic emission lines. We are determining the consequences of adding water to the samples in comparison to dry material.

In addition, we are examining the particles (atomic and molecular) and particulates generated from laser irradiation of $\mathrm{NaNO}_{3}$, observations which are important for ICP applications and concerns for exposure of workers and the environment near laser analysis of materials. We have discovered that in addition to melted, hydrodynamically sputtered material (droplets) and spalled $\mathrm{NaNO}_{3}$ crystallites, a form of smoke is observed. These smoke particles appear when ablation of $\mathrm{NaNO}_{3}$ is performed in $1 \mathrm{~atm}$ of air or $1 \mathrm{~atm}$ of Ar; in vacuum they are completely missing. They consist of aggregates of fairly uniformly sized $(1 \mu \mathrm{m})$ spheres and the aggregates tend to be ball shaped, also. We are currently trying to determine the chemical composition of the different types of particulates being produced. 


\section{Publications with cited DOE Sponsorship:}

R. L. Webb, L. C. Jensen, S. C. Langford, and J. T. Dickinson, "Interactions of wide bandgap single crystals with $248 \mathrm{~nm}$ excimer laser radiation: I. MgO", J. Appl. Phys. 74, 2323 (1993).

R. L. Webb, L. C. Jensen, S. C. Langford, and J. T. Dickinson, "Interactions of wide bandgap single crystals with $248 \mathrm{~nm}$ excimer laser radiation: II. NaCl", J. Appl. Phys. 742338 (1993)

J. T. Dickinson, L. C. Jensen, R. L. Webb, M. L. Dawes, and S. C. Langford, "Interactions of wide bandgap single crystals with $248 \mathrm{~nm}$ excimer laser radiation: III. the role of cleavage-induced defects in MgO," J. Appl. Phys. 743758 (1993).

J. T. Dickinson, L. C. Jensen, R. L. Webb, M. L. Dawes, and S. C. Langford, "Mechanisms of excimer laser ablation of wide bandgap materials: the role of defects in single crystal MgO," MRS Symposium Proceedings 285, 131 (1993).

J.T. Dickinson, Jaw-Jung Shin, W. Jiang, and M.G. Norton, "Neutral and ion emissions accompanying pulsed excimer laser irradiation of polytetrafluroethylene (PTFE)," J. Appl. Phys. 74, 4729 (1993).

J. T. Dickinson, L. C. Jensen, R. L. Webb, and S. C. Langford, "Laser ablation of wide band-gap materials: The role of defects in single crystal MgO," submitted to the Proceedings of the Conference on Laser Ablation (COLA) 1993.

J. Thomas Dickinson, "The role of defects in the laser ablation of wide bandgap materials," submitted to Nucl. Instrum. Methods Phys. Res. B.

J. T. Dickinson, Jaw-Jung Shin, W. Jiang, and M. G. Norton, "Evidence for unzipping reactions in the laser ablation of polytetrafluoroethylene (PTFE)," submitted to Nucl. Instrum. Methods Phys. Res. B.

J. Thomas Dickinson, L. C. Jensen, R. L. Webb, and S. C. Langford, "Laser ablation studies relevant to thin film deposition," submitted to ICAM-MRS Proceedings.

J. Thomas Dickinson, L. C. Jensen, R. L. Webb, J-J. Shin, and S. C. Langford, "The role of defects in the laser ablation of wide bandgap materials," submitted to SPIE Proceedings.

J. T. Dickinson, L. C. Jensen, and R. L. Webb, "Photoluminescence imaging of mechanically produced defects in MgO," submitted to J. Non-Cryst. Solids.

\section{Invited Talks:}

"Laser Ablation of Wide Bandgap Materials," MRS Fall Meeting, Boston, December, 1992.

"The Role of Defects in Laser Ablation of Transparent Dielectrics," Dept. of Chemical Engineering, University of Washington, January, 1993.

"Mechanical Consequences of Laser Bombardment of Insulators", Workshop on Dynamic Response of Materials to Energy Deposition, Los Alamos, January, 1993.

"The Detection of Crazing and Microcracking in Strained Polymers", Dow Co., February, 1993. 
"The Role of Defects in Laser Ablation of Transparent Dielectrics," Dept, of Nuclear Engineering. University of Michignn, February, 1993.

"Laser Ablation of Wide Bandgap Materials", International Workshop on Laser-Materials Interactions, Oak Ridge, April, 1993.

"Imaging Effects in Studies of Laser Ablation of Wide-Bandgap Materials," Scanning Microscopy 1993, L.os Angeles, May, 1993.

"Fracto-Emission from Polymer Interfaces," ACS Symposium on Adhesion, Chicago, August, 1993.

"Deformation and Fracture Induced Luminescence Phenomena in Polymers," ACS Symposium on Polymers, Chicago, August, 1993.

"Laser-Surface Interactions Relevant to Laser-Ablation Deposition", 3rd International Conference on Advanced Materials-MRS. Tokyo, Japan, August, 1993.

"Mechanisms of Laser Desorption from MgO," International Confererence on Radiation Effects on Insulators, Nagoya, Japan, September, 1993.

"Transient Electrical Measurements of Adhesive Failure," 3M Co., October, 1993.

"Fracto-Emission Studies in Polymers," Department of Materials Science, University of Michigan, October, 1993.

"The Role of Defects in Laser Interactions with Wide Bandgap Materials," Department of Materials Science, Michigan State University, October, 1993.

"The Role of Defects in Laser Interactions with Wide Bandgap Materials," Boulder Laser Damage Conference, October, 1993.

"New Studies of Excimer Laser Ablation of Wide Bandgap Materials," LEOS93, San Jose, CA, November, 1993

"Imaging the Consequences of Mechanical and Laser Stimulation of Materials," International Symposium on Fracture and Strength of Glass and Ceramic Materials, PACRIM 1993, Honolulu, Nov., 1993.

\section{To be presented:}

"Excimer Laser Interactions with Wide Bandgap Materials," LASER93, Lake Tahoe, Dec. 1993.

"New Studies in Fracture and Laser Ablation," 10 days of Lectures at TU and FU, Berlin, Germany, Spring, 1994.

"Consequences of UV Laser Irradiation of Glasses," Gordon Conference on Glass, June, 1994.

"Excimer Laser - Wide Band Gap Materials Interactions: Ablation Mechanisms", Gordon Conference on Laser Ablation, August, 1994. 

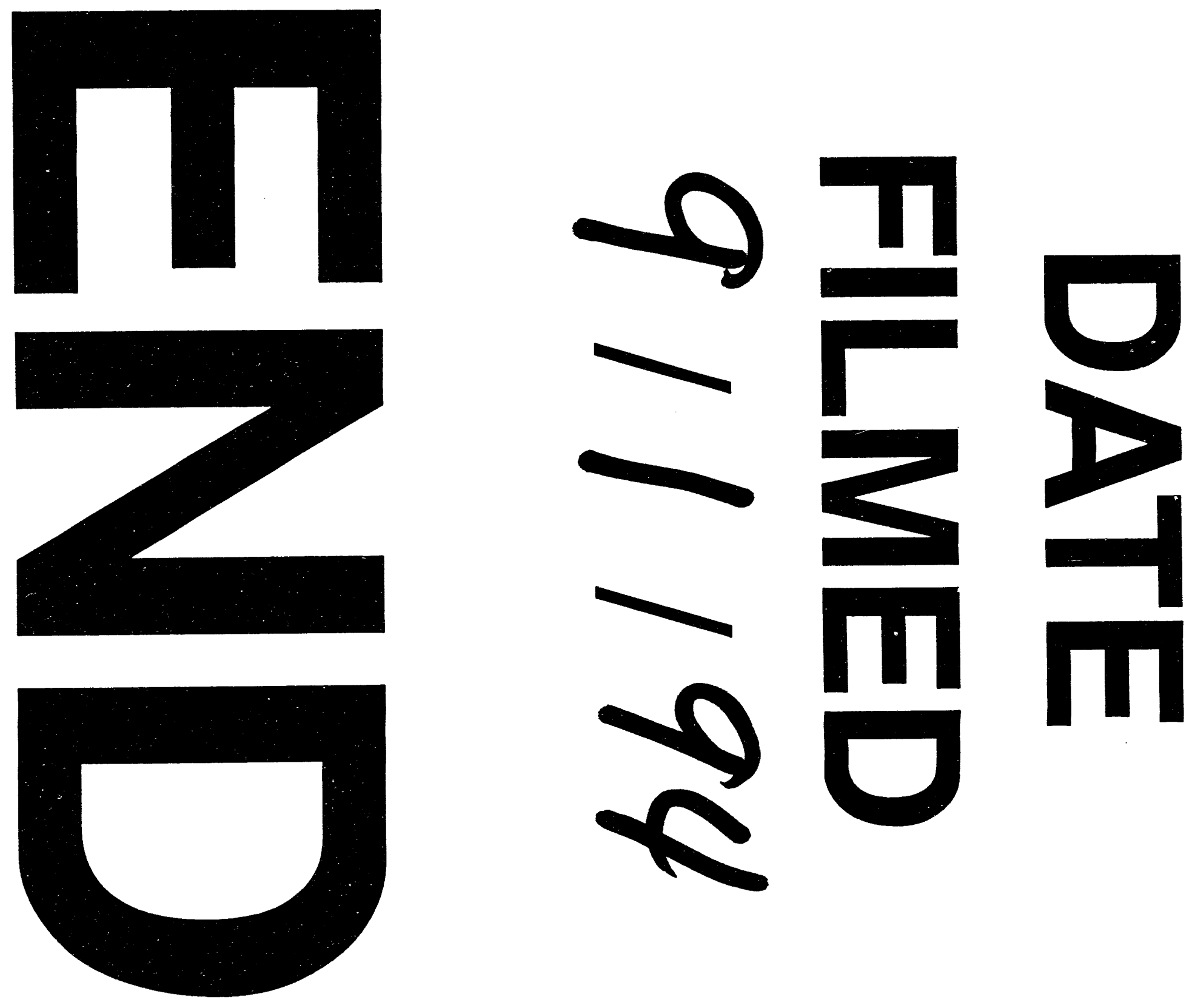
